\title{
DAMAGE DETECTION IN COMPOSITE MATERIALS USING LAMB WAVE METHODS
}

\author{
Seth S. Kessler ${ }^{*}$ and S. Mark Spearing \\ Department of Aeronautics and Astronautics, Massachusetts Institute of Technology, Cambridge, MA 02139, USA \\ Constantinos Soutis \\ Department of Aeronautics, Imperial College of Science Technology and Medicine, London SW7 2BY, UK
}

*Corresponding author: Email address - sskess@ mit.edu (S.S. Kessler). Fax - 630-214-8749 


\section{ABSTRACT}

Cost-effective and reliable damage detection is critical for the utilization of composite materials. This paper presents part of an experimental and analytical survey of candidate methods for in-situ damage detection of composite materials. Experimental results are presented for the application of Lamb wave techniques to quasi isotropic graphite/epoxy test specimens containing representative damage modes, including delamination, transverse ply cracks and through-holes. Linear wave scans were performed on narrow laminated specimens and sandwich beams with various cores by monitoring the transmitted waves with piezoceramic sensors (PZT). Optimal actuator and sensor configurations were devised through experimentation, and various types of driving signals were explored. These experiments provided a procedure capable of easily and accurately determining the time-of-flight of a Lamb wave pulse between an actuator and sensor. Lamb wave techniques provide more information about damage presence and severity than previously tested methods (frequency response techniques), and provide the possibility of determining damage location due to their local response nature. These methods may prove suitable for structural health monitoring applications since they travel long distances and can be applied with conformable piezoelectric actuators and sensors that require little power.

Keywords: Polymer-matrix composites; Damage detection; Non-destructive testing; Lamb waves; 


\section{INTRODUCTION}

\subsection{Health Monitoring of Composite Structures}

Structural Health Monitoring (SHM) has been defined in the literature as the "acquisition, validation and analysis of technical data to facilitate life-cycle management decisions.” [1] More generally, SHM denotes a system with the ability to detect and interpret adverse "changes" in a structure in order to improve reliability and reduce life-cycle costs. The greatest challenge in designing a SHM system is knowing what "changes" to look for and how to identify them. The characteristics of damage in a particular structure plays a key role in defining the architecture of the SHM system. The resulting "changes," or damage signature, will dictate the type of sensors that are required, which in-turn determines the requirements for the rest of the components in the system. The present research project focuses on the relationship between various sensors and their ability to detect "changes" in a structure's behavior.

The aerospace industry has one of the highest payoffs for SHM since damage can lead to catastrophic (and expensive) failures, and the vehicles involved have regular costly inspections. Currently $27 \%$ of an average aircraft's life cycle cost is spent on inspection and repair [2]; a figure that excludes the opportunity cost associated with the time the aircraft is grounded. These commercial and military vehicles are increasingly using composite materials to take advantage of their excellent specific strength and stiffness properties, fatigue performance, as well their ability to reduce radar cross-section and "part-count". The disadvantage, however, is that composite materials present challenges for design, manufacturing, maintenance and repair over metallic parts since they tend to fail by distributed and interacting damage modes [3, 4]. Furthermore, damage detection in composites is more difficult than in metallic structures due to the anisotropy of the material, the conductivity of the fibers, the insulative properties of the matrix, and the fact that much of the damage often occurs beneath the top surface of the laminate and is therefore not readily detectable (barely visible impact damage, or BVID). Currently successful composite non-destructive testing (NDT) techniques for small laboratory specimens, such as Xradiographic detection (penetrant-enhanced X-ray) and hydro-ultrasonics (C-scan), are impractical for in-service inspection of large components and integrated vehicles. It is clear that new reliable approaches for damage detection in composites need to be developed to ensure that the total cost of ownership of critical structures does not become a limiting factor for their use. 


\subsection{Survey of Lamb Wave Methods}

Several techniques have been researched for detecting damage in composite materials, however Lamb wave methods have recently re-emerged as a reliable way to locate damage in these materials [5-7]. These techniques have been implemented in a variety of fashions in the literature, including the use of separate actuators and sensors to monitor transmitted waves and/or reflected waves, and multipurpose patches which both actuate and sense. Each of these techniques offers their own unique advantages in detecting certain types of damage with various levels of analytical complexity. This section presents a sample of the most common applications of Lamb wave methods presented in the literature.

Perhaps the earliest recognition of Lamb waves as a means of damage detection came in 1960 by Worlton of the General Electric Company [8]. His report investigated the dispersion curves of aluminum and zirconium to describe analytically the characteristics of the various modes that would pertain to nondestructive testing applications. The motions and velocities of the interior particles are formulated, and the effects of holes and thickness variations are discussed. In the following decade Lamb wave techniques were examined by several investigators, and then the first potential aerospace application was introduced by Demer and Fentnor of the Hughes Aircraft Company [9]. In their work they cite ultrasonic wave testing as one of the most reliable forms of nondestructive testing, and that Lamb waves were emerging as one of the best ways to infer information about the medium's density, elastic properties and thickness. They did however acknowledge the fact that Lamb waves were not readily understood nor easily interpreted. The particular work performed at Hughes focused on Lamb wave propagation in metallic thin sheets and elongated cylindrical forms. Fatigue cracks were located in stainless-steel and aluminum rods by recording the time-of-flight and attenuated amplitude of the received ultrasonic signal. Similar experiments were performed on glass, ceramic and plastic specimens with the objective of examining the effects of liquid penetration.

During the late 1980's and early 1990's work began on the application of Lamb waves to composite materials. Research conducted at NASA by Saravanos demonstrated, both analytically and experimentally, the possibility of detecting delamination in composite beams using Lamb waves [10, 11]. Similar conclusions were drawn by Percival and Birt [12-14] at the Defense \& Evaluation Research Agency, UK, who began focusing their work on the two fundamental Lamb wave modes, which will be described further in the following section. Detection of other forms of damage in composite materials was also investigated by Seale [15], who examined 
fatigue and thermal damage, and Tang [16] who observed the sensitivity of Lamb wave propagation to fiber fracture. Work was also performed on composite sandwich plates subjected to impact damage by Rose [17] and Osmont [18].

The most successful work to date of using Lamb waves for damage detection has been performed by two separate groups at Imperial College. Since the mid-1990's, Cawley's group has been working to optimize the generation of directional Lamb waves $[19,20]$. To allow the implementation of Lamb waves on a real structure, they have been developing flexible, cheap Polyvinylidenedifloride (PVDF) transducers in order to both generate and detect waves. Cawley's work utilizes interdigital transducers to generate highly focused and directional waves without higher mode interference, and they have inspected various metallic specimens with encouraging results. Soutis's group in the Aeronautics department has focused more on the sensor placement and signal processing issues [21-23]. They have chosen to use Lead-Zirconate-Titanate (PZT) actuators and sensors over PVDF since they require a factor of ten less voltage to generate Lamb waves, however they are not conformable. The most complete work from this group can be found in Valdez's PhD thesis [24]. During the course of his work he performed many experiments on quasi-isotropic graphite/epoxy composite specimens, pulsing them with Lamb waves in various configurations to detect delaminations. He also simulated the propagation of Lamb waves in plates using a finite element code. Much of the research presented in this paper follows Valdez's work, extending it to various other types of damage, to sandwich structures, and an attempt to optimize the testing procedure and setup. The following section will discuss the fundamentals and mathematics of Lamb wave propagation.

\section{DESCRIPTION OF LAMB WAVES}

Lamb waves are a form of elastic perturbation that can propagate in a solid plate with free boundaries [25, 26]. This type of wave phenomenon was first described in theory by Horace Lamb in 1917, however he never attempted to produce them [27]. There are two groups of waves, symmetric and anti-symmetric, that satisfy the wave equation and boundary conditions for this problem, and each can propagate independently of the other. A graphical representation of these two groups of waves can be seen in Figure 1. The present work utilizes PZT piezoelectric patches to excite the first anti-symmetric Lamb wave $\left(\mathrm{A}_{0}\right.$ mode). This wave was chosen since it can propagate long distances with little dispersion, and no higher modes are present to clutter the resulting response waves [24]. The fundamental way to describe the propagation of a Lamb wave in a particular material is with their 
dispersion curves, which plot the phase and group velocities versus the excitation frequency. The derivation of these curves begins with the solution to the wave equation for the anti-symmetric Lamb wave as seen in Equation 1:

$$
\frac{\tan \left(\overline{\mathrm{d}} \sqrt{1-\zeta^{2}}\right)}{\tan \left(\overline{\mathrm{d}} \sqrt{\xi^{2}-\zeta^{2}}\right)}+\frac{\left(2 \zeta^{2}-1\right)^{2}}{4 \zeta^{2} \sqrt{1-\zeta^{2}} \sqrt{\xi^{2}-\zeta^{2}}}=0
$$

where the non-dimensional parameters are:

$$
\xi^{2}=\frac{c_{t}^{2}}{c_{l}^{2}}, \zeta^{2}=\frac{c_{t}^{2}}{c_{\text {phase }}^{2}}, \overline{\mathrm{d}}=\frac{\mathrm{k}_{\mathrm{t}} t}{2}
$$

These velocities can be defined by Lamé's constants:

$$
\begin{gathered}
\mu=\frac{\mathrm{E}}{2(1+\mathrm{v})}, \lambda=\frac{\mathrm{Ev}}{(1-2 v)(1+v)} \\
c_{t}^{2}=\frac{\mu}{\rho}, c_{l}^{2}=\frac{(\lambda+2 \mu)}{\rho}, \mathrm{k}_{\mathrm{t}}=\frac{\omega}{c_{t}}
\end{gathered}
$$

Substituting these equalities into the non-dimensional parameters yields:

$$
\begin{aligned}
& \xi^{2}=\frac{\mu}{(\lambda+2 \mu)}=\frac{1-2 v}{2-2 v}, \zeta^{2}=\frac{\mu}{\rho c_{\text {phase }}^{2}}=\frac{E}{2 \rho(1+v) c_{\text {phase }}^{2}} \\
& \overline{\mathrm{d}}=\frac{\omega t}{2 c_{t}}=\frac{\omega t}{2} \sqrt{\frac{\rho}{\mu}}=\frac{\omega t}{2} \sqrt{\frac{2 \rho(1+v)}{E}}
\end{aligned}
$$

Finally, Equation 5 can be substituted into Equation 1 to be solved numerically. For a given material, the Young's Module $\boldsymbol{E}$, Poisson Ratio $\boldsymbol{v}$, and the density $\rho$ are known, and the Phase velocity $c_{\text {phase }}$ is the dependent variable being solved. The independent variable being iteratatively solved for is the frequency-thickness product, where $\omega$ is the driving frequency in radians/sec. An example of a phase velocity dispersion curve for the first anti-symmetric Lamb wave using the material properties from the specimens used in the present research can be seen in Figure 2. The other useful plot is the group velocity dispersion curve, which can easily be derived from the phase velocity curve using Equation 6:

$$
\begin{aligned}
& k=\frac{2 \pi}{\lambda_{w}} \text { (wave \#), } \lambda_{w}=\frac{c_{\text {phase }}}{f}(\text { wavelength }) \\
& c_{\text {group }}=c_{\text {phase }}+\frac{\partial c_{\text {phase }}}{\partial k} k=\frac{c_{\text {phase }}}{1-\frac{f}{c_{\text {phase }}} \cdot \frac{\partial c_{\text {phase }}}{\partial f}}
\end{aligned}
$$


An example of a group velocity dispersion curve, again using the material properties from the present research can be seen in Figure 3. The equations presented here are intended for isotropic materials, however it has been shown in the literature that the $A_{o}$ is fairly invariant to the layup of a composite material, and can be closely approximated by using the bulk laminate properties [13]. Finite element techniques have been used by other researchers in the literature to more accurately determine the wave velocities in composite materials $[12,15]$.

The group velocity of a Lamb wave produced by a piezoelectric patch driven at a particular frequency can easily be verified in a control (undamaged) specimen by measuring the time-of-flight (TOF) in an oscilloscope between two sensors of known separation. This information can then be used to locate damaged areas along a specimen, without using any analytical models, by observing the disturbed wave between the sensor and actuator. The lamb wave's group velocity essentially varies by a similar equality to that of a structure's resonant frequency, as $(E / \rho)^{1 / 2}$, where $E$ is modulus and $\rho$ is density, so as a wave travels across an area of reduced stiffness it will slow down. Another phenomenon associated with damage is analogous to traveling acoustical waves; upon reaching a region of dissimilar wave speed, a portion of the wave is reflected proportionally to the difference in their stiffness and density. From these two pieces of information, good correlation with damage location and magnitude can be determined. Several experiments were performed in the present research to establish the effectiveness of this procedure in determining the damage present in a composite laminate.

\section{OPTIMIZATION}

There is currently no standard or even a best-practice precedent for damage detection via Lamb wave testing. Several procedures have been developed in the literature, each with valuable characteristics, and each with some degree of arbitrariness. The goal of the first part of the present research was to determine experimentally and analytically what effects various parameters have on the sensitivity of damage detection. These parameters can be divided into three categories: actuator and sensor geometry, actuation pulse, and specimen properties. The first of these categories was not explored thoroughly in this research. A few shapes of piezoceramic patches were used to produce Lamb waves, and as expected waves propagated parallel to each edge, i.e. longitudinally and transversely for a rectangular patch and circumferentially from a circular piezo. Several other researchers have examined the effects of piezo dimensions on their actuation, thus it was not of particular interest for the present research [28, 29]. 
The second set of variables explored was the actuation pulse parameters. These included the pulse shape, amplitude, frequency and number of cycles to be sent during each pulse period. These parameters were varied experimentally on a control specimen to observe their effect on the Lamb waves generated. Two PZT piezoceramic patches were attached to either end of the specimen, one connected to an arbitrary function generator and the other to an oscilloscope as seen in Figure 4. For each pulse shape, the various parameters were changed independently as the transmitted wave was observed in the oscilloscope. The shapes that produced the best results were then compared in Matlab ${ }^{\mathrm{TM}}$ by using their power-spectral-density (PSD) plots. Similarly the effect of the number of cycles per period for the different shaped signals was observed in the PSD plots by comparing the energy dedicated to the principal driving frequency. The more energy dedicated to the desired driving frequency, the stronger the Lamb wave and the more accurate the wave speed calculation, and hence the more sensitive and reliable the damage detection capability.

The final component of the optimization analysis mathematically quantified the significance of the specimens' geometric and material properties. The Lamb solution for the wave equation was used to plot the dispersion curves for each proposed specimen configuration as described in the previous section. The material constants for the composite laminates to be analyzed were calculated by classical laminated plate theory, and then inputted into the mathematical model [30]. Using the same model code, each material constant such as the tensile modulus, Poisson ratio and density were modified independently, and the effect on the dispersion curves was documented. Since the dependent variable in this mathematical model was the frequency-thickness product, changes in specimen thickness were easily quantifiable since they proportionally shifted the plot along the x-axis. From the optimization experiments and analysis an effective test method was determined, which was then used to detect damage in several test specimens.

\section{EXPERIMENTAL PROCEDURE}

\subsection{Narrow Coupon Tests}

Following a building block approach [31], the first set of experiments was conducted on narrow composite coupons. The laminates used for this present research were manufactured during previous research that explored frequency response methods as a means of damage detection, and were re-used to compare directly the effectiveness of the two methods [32]. The specimens were 25 x $5 \mathrm{~cm}$ rectangular $[90 / \pm 45 / 0]_{\mathrm{s}}$ quasi-isotropic laminates of the 
AS4/3501-6 graphite/epoxy system, which were clamped on one end to match the boundary conditions from the previous research (however experimentation proved that the boundary conditions around the frame of the specimen had no effect on the Lamb wave traveling between two piezoceramic patches). Three PZT piezoceramic patches were affixed to each specimen, as shown in Figure 4, using $3 \mathrm{M}$ ThermoBond ${ }^{\mathrm{TM}}$ thermoplastic tape so that they were firmly attached during testing, but could be removed afterwards to recover the specimens for future tests. The PZT was cut into $2 \times 0.5 \mathrm{~cm}$ patches so that the longitudinal wave would be favored over the transverse one, and three patches were used on each specimen to actuate and accurately measure the transmitted and reflected waves. Both the actuation and the data acquisition were performed using a portable NI-Daqpad ${ }^{\mathrm{TM}} 6070 \mathrm{E}$ data acquisition board, and a laptop running Labview ${ }^{\mathrm{TM}}$ as a virtual controller. A Labview ${ }^{\mathrm{TM}}$ VI-file was created which would load an arbitrary waveform from Matlab ${ }^{\mathrm{TM}}$ and output it at the desired frequency and amplitude, while simultaneously acquiring data on four channels at 600,000 samples per second. The first channel, which served as the trigger for all of the channels, was connected to the output channel and actuating PZT, two others were connected to the sensing piezoceramic patches, and the final channel was connected to a PZT sensor not attached to the specimen to serve as a control channel in order to zero out drift. A single pulse of the optimal signal found in the previous section, shown in Figure 5, was sent to the driving PZT patch to stimulate an $\mathrm{A}_{0}$ mode Lamb wave, and concurrently the straininduced voltage outputs of the other two patches were recorded for $1 \mathrm{~ms}$ to monitor the wave propagation.

The resulting data was then passed to Matlab ${ }^{\mathrm{TM}}$ where the drift was filtered out and the waveforms could be compared and analyzed within two specialized toolboxes. In the signal processing toolbox the waves could be easily superimposed, and a built-in peak detector was used to determine accurately the time-of-flight for each signal, and the delay in time of arrival between two specimens. Subsequently, in the wavelet toolbox a DB3 wavelet was used to decompose the data into its frequency components. By plotting the magnitude of the wavelet coefficient at the peak driving frequency, the energy remaining from the input signal could be compared [33]. This procedure was carried out for two of each specimen type at the driving frequency of $15 \mathrm{kHz}$.

As with the previous research on frequency response methods [32], various types of damage were introduced to the specimens. In the first group, $6.4 \mathrm{~mm}$ diameter holes were drilled into the center of each specimen as a stress concentration. The next group was compressively loaded in a 4-point bending fixture until audible fiber fracture damage was heard, and the third was cyclically loaded in the same fixture for 2000 cycles at $80 \%$ of this load with an $\mathrm{R}$ ratio of -1 to create matrix cracks. The next two groups of specimens were delamination specimens 
which were introduced by two methods: one used a thin utility blade to cut a 5 x $2.5 \mathrm{~cm}$ slot in one side, and the other with a Teflon strip cured into the center mid-plane of the laminate. After the damage was introduced into each specimen, an x-ray radiograph was taken using a die-penetrant to help document the type, degree and location of the damage as shown in Figure 6.

\subsection{Sandwich Coupon Tests}

Analogous experiments were performed on sandwich coupons to that of the narrow laminates in order to test the effect of various types of core materials on the propagation of Lamb waves. Four different cores were used: low and high density (referred to as LD and HD) aluminum honeycomb, Nomex ${ }^{\mathrm{TM}}$, and Rohacell ${ }^{\mathrm{TM}}$. Each specimen contained two facesheets identical to the undamaged laminates in the previous section surrounding a $2 \mathrm{~cm}$ thick core, which were adhered using FM-123 film adhesive in a secondary curing process. Two controls and two damaged specimens of each type were manufactured for testing. In the damaged specimens, a $5 \mathrm{x} 2.5 \mathrm{~cm}$ piece of Teflon was placed between the adhesive and the core in a central $2.5 \mathrm{~cm}$ region during the cure so that the facesheet would not bond to the honeycomb to simulate a delamination. An additional specimen was also manufactured with the high density aluminum core that had a $2 \mathrm{~cm}$ diameter circular piece of Teflon placed between the layers on either side so that it was indistinguishable from the controls by sight. This specimen was used for a "blind test" of the proposed Lamb wave damage detection method, where it was tested alongside the two control specimens to determine which had the artificial flaw. The test setup and data analysis procedure for the sandwich beam experiments were identical to that of the thin specimens with the exception of the driving frequency, which was determined to be more effective at $50 \mathrm{kHz}$ for these tests.

\section{RESULTS}

\subsection{Optimization Results}

\subsubsection{Pulse Frequency:}

The dispersion curves show the relationship between the phase velocity and pulse frequency. At lower frequencies, fewer Lamb modes are excited so the response signal is more distinguishable, and the velocity is slower so there is more time separating the sent and received signals making any changes more distinguishable. At these 
lower frequencies however, the dispersion curves have steep slopes and thus are very sensitive to small variations in frequency making it difficult to predict the time-of-flight. At higher frequencies, when more modes are present, the slope then tends to flatten out with the consequence of a shorter wave pulse carrying less or more compressed information on the damage. For the experiments performed during this present research, $15 \mathrm{kHz}$ was chosen for the thin laminate tests, and $50 \mathrm{kHz}$ for the sandwich beam tests as the optimal testing points. These frequencies were based on their slope and location on the dispersion curves, evidence from previous research suggesting these frequencies for specimen of similar geometries, and brief experimentation using a function generator to determine the maximum response amplitude for a range of driving frequencies.

\subsubsection{Pulse Amplitude:}

Increasing the driving voltage proportionately increases the magnitude of the Lamb wave strain. In these experiments, driving the piezopatches at an amplitude of 5-10V produced a $10-25 \mathrm{mV}$ response due to the wave sensed by the PZT patch. Increasing the amplitude also increases the signal to noise ratio to yield a clearer signal, since the static noise received by the PZT patch is usually in the $1-5 \mathrm{mV}$ range. Higher voltage however also tended to increase the drift in the signal, which deteriorated the resolution capabilities of the data acquisition system. Also, a potential SHM system should be as low power as possible. The optimal driving voltage was therefore chosen to be $5 \mathrm{~V}$ for these experiments.

\subsubsection{Number of cycles:}

The number of cycles of a periodic function to actuate the piezopatches with, is one of the more complicated decisions to be made for Lamb wave techniques. The FFT of a continuous sine wave would yield single peak at the driving frequency, however for a few finite cycles, the FFT appears as a Gaussian curve with a peak at the driving frequency. Thus, the more periods of a wave sent into a driving pulse, the narrower the bandwidth and the less dispersion. The problem in a short specimen though, is the more periods of a wave in the pulse, the less time between last sent signal and the first reflected one, so the response is more difficult to interpret. An appropriate number of cycles can be determined by the maximum number of waves that can be sent in the time it takes for the lead wave to travel to the sensing PZT patch. It is also convenient to use intervals of half cycles so that the sent sinusoidal pulse becomes symmetric. Research from the literature has used signals varying from 3.5 to 13.5 
cycles per actuating pulse [17-24]. Since the specimens in the current research are relatively short, few cycles could be actuated without disturbing the received signal thus 3.5 cycles were used to drive the piezoceramic actuators.

\subsubsection{Pulse Shape:}

Of the signal shapes that were analyzed and experimented, pure sinusoidal shapes appear to excite Lamb wave harmonics the most efficiently, since they are periodic, smooth and have comparatively quick rise times to their peak amplitude as compared to a parabolic shape. A Hanning window (approximated by a half-sine wave multiplied over the pulse width) helps to narrow the bandwidth further to focus the maximum amount of energy into the desired actuating frequency with the least "spill-over" from neighboring frequencies.

\subsubsection{Material Properties:}

The relationship between the material properties of a specimen and the speed of the propagating Lamb wave is quite complex, however an understanding is necessary to design an appropriate damage detection test. To first order, the wave velocity increases with the square root of the modulus, i.e. an increase in modulus slightly speeds the wave velocity. An increase in the density would have the opposite effect however by slowing wave velocity, as it appears in all the same terms as the modulus but on the reciprocal side of the divisor. The effect of the Poisson's ratio is probably the most complicated, as it appears in the most terms, however to first order, small changes seem to have little to no effect on the wave velocity. The most straightforward parameter is the thickness of the specimen, which has a linear relationship with the Lamb wave velocity. The thicker the specimen the quicker the speed and the higher the dispersion rate for a given driving frequency.

\subsection{Experimental Testing Results}

There were two sets of results obtained for both the thin coupons and the narrow sandwich beams. The first set of results included the virgin time traces of voltage from the PZT sensor at the far end of the specimen. For the thin coupons, $1 \mathrm{~ms}$ of data was taken and the average peak voltage was around $20 \mathrm{mV}$. The time traces for one of each type of specimen along with a superimposed control specimen are shown in Figure 7. Similarly, $500 \mu$ s of data was taken for the sandwich beams with an average peak voltage of around $10 \mathrm{mV}$. For these specimens, time traces of each control beam are plotted against their delaminated complement in Figure 8. In each of these plots, a "bleed-through" portion of the sent signal leaking across the data acquisition board can be seen at the beginning of 
the time trace. Since the channels were all triggered at the $5 \mathrm{~V}$ peak voltage, exactly half of the sent signal is visible so this became a convenient way to measure the time-of-flight. The second set of results for each specimen group was the outcome of the wavelet decomposition. For each specimen, the "bleed-through" portion of the signal was filtered out, and the wavelet coefficient magnitude of the dominant frequency $(15 \mathrm{kHz}$ for the thin coupons and 50 $\mathrm{kHz}$ for the beams) was plotted over time. For the thin coupons, Figure 9 compares these coefficients, and thus the transmitted energy, for one of each type of specimen. Finally, Figure $\mathbf{1 0}$ displays the coefficient magnitude results for the "blind test," comparing the two high density aluminum core control specimens with one known and one unknown damaged specimen.

\section{DISCUSSION}

\subsection{Experimental Procedure Optimization}

There were two important sets of guidelines obtained from the optimization portion of this research. The first set was the analytical trade studies performed to predict the effectiveness of Lamb wave methods in different applications. Using the mathematical formulations derived by Lamb, the effects of material constants and specimen geometry were determined. By entering the material properties for a particular application the resulting dispersion curves provide a range of potential wave velocities for the $A_{0}$ mode driven at different frequencies. If the characteristic wave velocity for a material is too fast to be reasonably acquired, then this method is not suitable. Also, with knowledge of the effects of various damage types on the stiffness of a particular material, the resolution of change for the resultant signal, or "observeability" can be predicted in order to determine the detection limitations with respect to flaw size for a given data acquisition capability. This procedure was conducted prior to the experimental procedure of this research, and it was determined that graphite/epoxy composite material was a good candidate for Lamb wave methods, and that with the detection capabilities of the data acquisition system that a reasonable change in stiffness $(5-10 \%)$ could be resolved.

The second guideline provided an experimental and analytical determination of the optimal testing configuration to be applied for a particular material, including the driving frequency, number of cycles, and the minimal required distance for placement of the actuators away from boundaries or features. Using the dispersion curves for a material, a range of potential driving frequencies can be selected based upon regions of smallest slope and driving capabilities while remaining below frequencies that would generate higher order waves. Next, 
experimentally these frequencies can be tuned using a function generator to find the optimal frequency in that range that produces the largest amplitude Lamb wave. There is then a trade between number of waves that can be sent in a pulse and the distance from abrupt features in the structure; as discussed previously the more waves the more energy that goes into the Lamb wave, however if features are too close to the actuator/sensor with many waves then changes in response signal may be obscured. For the experiments presented in this paper, it was determined that 15 $\mathrm{kHz}$ was the optimal driving frequency for the thin laminates and $50 \mathrm{kHz}$ for the sandwich beam specimen, using a signal of 3.5 sine waves since the specimen were relatively short. These sets of tools could be used in tandem by an engineer developing a SHM system for a vehicle to decide if the Lamb wave method would provide satisfying results for their application, and to determine the appropriate driving parameters to obtain the best damage detection resolution.

\subsection{Interpretation of Experimental Data}

There are generally five goals for damage detection, each of which is gained with increasing difficulty and complexity. The first is the determination of the presence of damage in a specimen. The second is an estimation of the extent of severity of the damage. The third goal is to be able to differentiate between various different types of damage. The fourth is to be able to calculate where the damage is located. The final is to estimate the size of the damage. It appears that Lamb wave methods carry enough information potentially to meet all of these goals with a strategically placed array of sensors and suitable processing codes, however the current scope of this research focuses on the first two goals.

The results from the narrow coupon tests clearly show the presence of damage in all of the specimens.

First of all, when the time traces of all of the control specimens were overlaid, there was a high degree of visible correlation, especially for the first half of the voltage time trace. The slight variation in the second half of the data can be attributed to the reflected signals returning from the far end of the specimen and passing under the PZT sensor again, which may encounter a slight cutting bias in the composite to cause a change in phase. Of the artificially damaged specimens, the Teflon-induced delamination was most easily quantified. When compared to the control specimens, these time traces appear at the same phase and frequency, only having been delayed about $55 \mu \mathrm{s}$

due to the damage. For the other types of damage the frequency often remained the same, however there was a large reduction in amplitude, and a large and varying change in phase. This time trace was reproducible within a single 
specimen, although would not be consistent across multiple specimens with identical forms of damage. This is due to the scatter and reflecting of the waves on the various feature of damage which may not be identical specimen to specimen, which makes a "damage signature" difficult to define. The most distinctly altered signal was that of the through-hole, having the same diameter as the actuator and sensor widths, which had the smallest voltage magnitude of all the specimens. The most obvious method to distinguish between damaged and undamaged specimens however is by regarding the wavelet decomposition plots. The control specimens retained over twice as much energy at the peak frequency as compared to all of the damaged specimens, and especially contained much more energy in the reflected waves. The loss of energy in the damaged specimens again is due to the dispersion caused by the micro-cracks within the laminate in the excitation of high-frequency local modes.

The sandwich beam results were more difficult to interpret, due to the damping nature of the cores significantly reducing the voltage generated by the PZT sensors. The high density aluminum core, which was the stiffest of the four tested, provided the clearest results; the other specimens yielded decreasing magnitude voltages as the stiffness decreased thus increasing the damping factor. There were two basic trends across all the specimens. The first was that the responses of the control specimens were larger than those that were delaminated for each core type. This is most likely due to the loss of energy of the wave in a local mode over the delaminated region. The second trend was the appearance of more reflected waves after the initial pulse in the time trace in the delaminated specimens, which again was probably due to other higher frequency modes being excited in the region of reduced thickness and dampening. Probably the most significant result of the present research was the "blind test." Four high density aluminum beam specimen were tested, one of which had a known delamination in its center, while of the remaining three specimens it was unknown which contained the circular disbond and which two were the undamaged controls. By comparing the four wavelet coefficient plots in Figure 10, one can easily deduce that the two control specimens are the ones with much more energy in the transmitted signals, while the third specimen (Control C) obviously has the flaw that reduces energy to a similar level to that of the known delaminated specimen. This test serves as a true testament to the viability of the Lamb Wave method being able to detect damage in at least simple structures. 


\subsection{Implementation of Lamb Wave Techniques in an SHM System}

Lamb wave techniques have good potential for implementation in an SHM system. These methods provide useful information about the presence, location, type, size and extent of damage in composite materials, and can be applied to a structure with conformable piezoelectric devices. The major disadvantage of this method is that it is active; it requires a voltage supply and function generating signal to be supplied. This can be complicated in a large structure, especially if the SHM system is to be implemented wirelessly; it has been suggested in the literature however that PZT can be actuated remotely using radio frequency waves [24]. Another difficult requirement is the high data acquisition rate needed to gain useful signal resolution. If a system is sampling at $0.5 \mathrm{MHz}$ from several sensors, a large volume of data will accumulate quickly; this implies the need for local processing. The data acquisition capabilities dictate the limitations of flaw size able to be resolved by a system using this method. In order to conserve power and data storage space, the Lamb wave method should most likely be placed into a SHM system in conjunction with another passive detection method, such as a frequency response method. The piezoelectric patches used to actuate the Lamb waves could passively record frequency response data until a certain threshold of change is surpassed, and then trigger the generation of Lamb waves to gain more specific data about the damaged region. Three to four piezoelectric multi-functioning actuator/sensor patches would be placed in the same vicinity in order to be able to triangulate damage location based upon reciprocal times of flight and reflected waves. The separation between sensing patches would depend on several parameters such as the material properties, damping characteristics and curvature of the structure, which for flat areas could be as large as 2 meters apart [24].

The detailed specifications of the Lamb wave method to be used for a particular application would be designed by the procedure described in the previous optimization section. Another useful detection capability arises from the fact that two different optimal driving frequencies were necessary for the thin laminates and the beam structures. This offers the possibility of having the ability to differentiate between damage within the laminate versus damage between the laminate and the core by discretely driving at two different frequencies. This procedure was not explored during the present research, however preliminary experimentation indicates that the potential of this procedure working exists. 


\section{CONCLUSIONS}

This paper has explored the optimization and application of Lamb wave methods to damage detection in composite materials. Several mathematical trade studies were preformed to observe the effect of various material constants and actuator driving parameters. Using these tools, an optimal configuration was selected for the experimental section of this research. For this optimal procedure, several narrow graphite/epoxy specimens were tested with various forms of pre-existing damage, such as delamination, matrix-cracks and through-thickness holes. Similar tests were also performed on narrow sandwich beams using cores of various densities and stiffness. These tests demonstrated the feasibility of detecting several types of flaws in representative composite structures, and this method was validated successfully by a "blind test" of several beam specimens. Lamb wave techniques have the shown the potential to provide more information than previously tested methods such as frequency response methods (FRM) since they are more sensitive to the local effects of damage in a material as compared to the global nature of FRM [32]. Similar to frequency response methods, their results are limited at higher frequencies, however their low frequency results should provide sufficient data to predict damage. The disadvantage of Lamb wave methods is that they require an active driving mechanism to propagate the waves, and the resulting data can be more complicated to interpret than for many other techniques. Overall however, Lamb wave methods have been found to be effective for the in-situ determination of the presence and severity of damage in composite materials, and hold the potential to locate damage due to their local response nature, which will be addressed in future research. Further experimentation will be aimed at testing two-dimensional and built-up structures using this technique, and the application of Lamb wave methods using a single multi-purpose actuator and sensor. Structural heath monitoring systems will be an important component in future designs of air and spacecraft to increase the feasibility of their missions, and Lamb wave techniques will likely play a role in these systems. 


\section{REFERENCES}

1. Hall S.R. "The Effective Management and Use of Structural Health Data." Proceedings of the $2^{\text {nd }}$ International Workshop on Structural Health Monitoring, 1999, 265-275.

2. Hall S.R. and T.J. Conquest. "The Total Data Integrity Initiative-Structural Health Monitoring, The Next Generation." Proceedings of the USAF ASIP Conference, 1999. $2^{\text {nd }}$ ed.

3. Bhat. N. "Delamination Growth in Graphite/Epoxy Composite Laminates Under Tensile Load." Massachusetts Institute of Technology, Cambridge, Ma, USA. 1993.

4. Wang S.S. "Delamination Crack Growth in Unidirectional Fiber-Reinforced Laminates under Static and Cyclic Loading." Composite Material: Testing and Design, ASTM STP (674), 1979, 642-663.

5. Bar-Cohen Y. "NDE of Fiber Reinforced Composite Materials -A Review." Materials Evaluation, v.44, 1986, 446-454.

6. Chang FK. "Structural Health Monitoring: A Summary Report." Proceedings of the $2^{\text {nd }}$ International Workshop on Structural Health Monitoring, Stanford, CA, September 8-10, 1999.

7. Giurgiutiu V., Jingjing B. and W. Zhao "Active Sensor Wave Propagation Health Monitoring of Bean and Plate Structures." Proceedings of the SPIE International Symposium on Smart Structures and Material, 2001.

8. Worlton D.C. "Experimental Confirmation of Lamb Waves a Megacycle Frequencies." Journal of Applied Physics, v.32, 1961, 967-971.

9. Demer L.J. and L.H. Fentnor. "Lamb Wave Techniques in Nondestructive Testing." International Journal of Nondestructive Testing, v.1, 1969, 251-283.

10. Saravanos D.A. and P.R. Heyliger. "Coupled Layerwise Analysis of Composite Beans with Embedded Piezoelectric Sensors and Actuators." Journal of Intelligent Material Systems and Structures, v.6, 1995, 350362.

11. Saravanos D.A., Birman V. and D.A. Hopkins. "Detection of Delaminations in Composite Beams using Piezoelectric Sensors." Proceedings of the $35^{\text {th }}$ Structures, Structural Dynamics and Materials Conference of the AIAA, 1994.

12. Percival W.J. and E.A. Birt. "A Study of Lamb Wave Propagation in Carbon-Fibre Composites." Insight: NonDestructive Testing and Condition Monitoring, v.39, 1997, 728-735.

13. Birt E.A. "Damage Detection in Carbon-Fibre Composites using ultrasonic Lamb Waves." Insight: NonDestructive Testing and Condition Monitoring, v.40, 1998, 335-339.

14. Badcock R.A. and E.A.Birt "The use of 0-3 Piezocomposite Embedded Lamb Wave Sensors for Detection of Damage in Advanced Fibre Composites." Smart Materials and Structures, v.9, 2000, 291-297.

15. Seale M.D., Smith B.T. and W.H. Prosser. "Lamb Wave Assessment of Fatigue and Thermal Damage in Composites." Journal of the Acoustic Society of America, v.103, 1998, 2416-2424.

16. Tang B. and E.G. Henneke. "Lamb Wave Monitoring of Axial Stiffness Reduction of Laminated Composite Plates." Materials Evaluation, v.47, 1989, 928-933.

17. Rose J.L., Hay T. and V.S. Agarwala. "Skin to Honeycomb Core Delamination Detection with Guided Waves." $4^{\text {th }}$ Joint DOD/FAA/NASA Conference on Aging Aircraft, 2000.

18. Osmont D., Devillers D. and F. Taillade. "A Piezoelectric Based healther Monitoring System for Sandwich Plates Submitted to Damaging Impacts." European Congress on Computational Methods in Applied Sciences and Engineering, 2000.

19. Monkhouse R.S.C., Wilcox P.D. and P. Cawley. "Flexible Interdigital PVDF Transducers for the Generation of Lamb Waves in Structures." Ultrasonics, v.35, 1997, 489-498.

20. Monkhouse R.S.C., Wilcox P.W., Lowe M.J.S., Dalton R.P. and P. Cawley. "The Rapid Monitoring of Structures using Interdigital Lamb Wave Transducers." Smart Materials and Structures, v.9, 2000, 304-309.

21. Valdez S.H.D. and C. Soutis. "Structural Integrity Monitoring of CFRP Laminates using Piezoelectric Devises." Proceedings of the European Conference on Composite Materials, 2000.

22. Valdez S.H.D. and C. Soutis. "Health Monitoring of Composites using Lamb Waves generated by Piezoelectric Devices." Plastics, Rubber and Composites, v.29, 2000, 475-481.

23. Valdez S.H.D. and C. Soutis. "A Structural Health Monitoring System for Laminated Composites." Proceedings of the $18^{\text {th }}$ Biennial Conference on Mechanical Vibration and Noise, 2001.

24. Valdez S.H.D. 'Structural Integrity Monitoring of CFRP Laminates using Piezoelectric Devices." Ph.D. thesis, Imperial College of Science Technology and Medicine, September 2000.

25. Viktorov I.A. Rayleigh and Lamb Waves, Physical Theory and Applications. Plenum Press, New York, 1967. 
26. Nayfeh A.H. Wave Propagation in Layered Anisotropic Media with Applications to Composites. v.39, Elsevier, Amsterdam, 1995.

27. Lamb H. "On Waves in an Elastic Plate." Proceedings of the Royal Society of London. Series A, Containing Papers of a Mathematical and Physical Character, v.93, n.651, 1917, 293-312.

28. Fripp M. "Distributed Structural Actuation and Control with Electrostrictors." SM thesis, Massachusetts Institute of Technology, June 1995.

29. Lively P. "Dynamic Structural Shape Estimation using htegral Sensor Arrays." SM thesis, Massachusetts Institute of Technology, June 2000.

30. Jones, R. M. Mechanics of Composite Materials. ${ }^{\text {nd }}$ ed, Taylor \& Francis, Blacksburg, VA, 1999.

31. "The Composite Materials Handbook MIL-17 Vol. 1" Guidelines for Characterization of Structural Materials." MIL-HDBK-1E, Department of Defense, 1999.

32. Kessler S.S., Spearing S.M., Atalla M.J., Cesnik C.E.S. and C. Soutis. "Structural Health Monitoring in Composite Materials using Frequency Response Methods." Proceedings of the SPIE International Symposium on Smart Structures and Material, 2001.

33. Lind R., Kyle S. and M. Brenner. "Wavelet Analysis to Characterize Non-Linearities and Predict Limit Cycles of an Aeroelastic System." Mechanical Systems and Signal Processing, v.15, 2001, 337-356. 


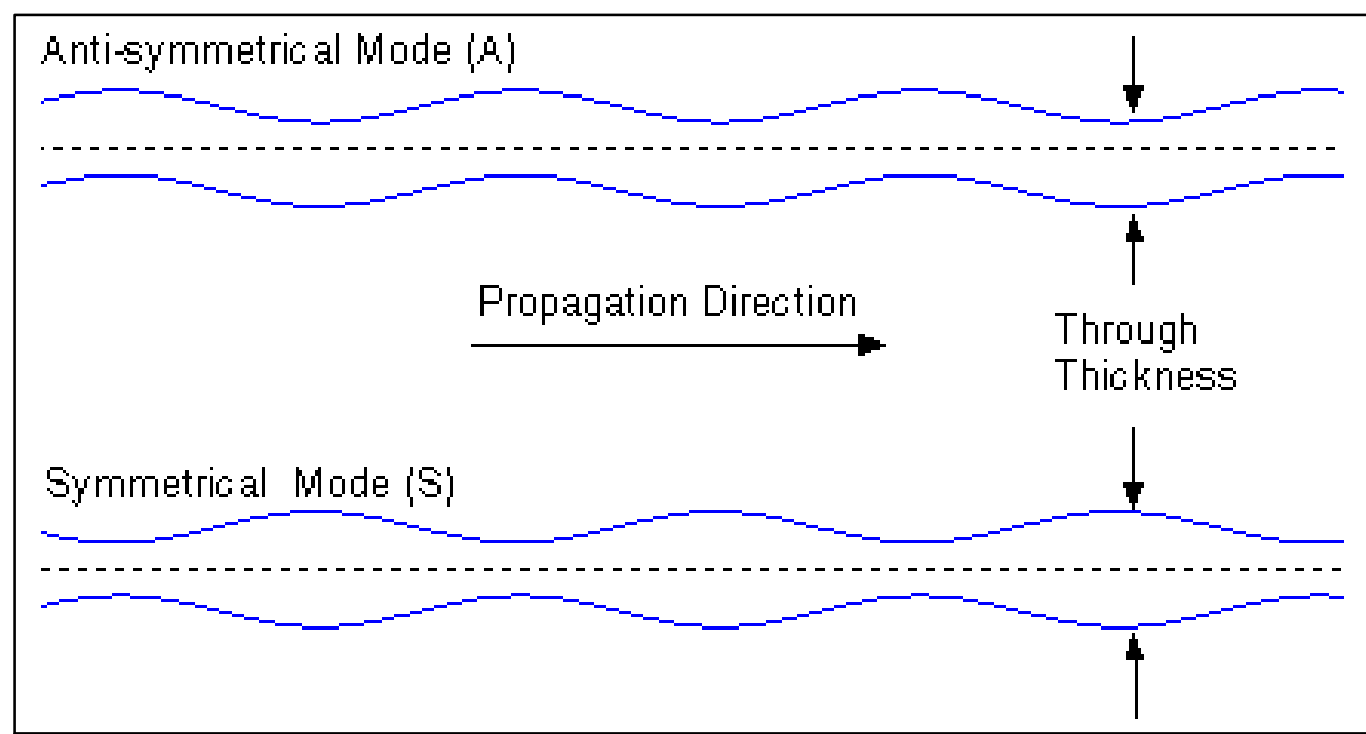

Figure 1: Graphical representation of $A$ and $S$ Lamb wave shapes

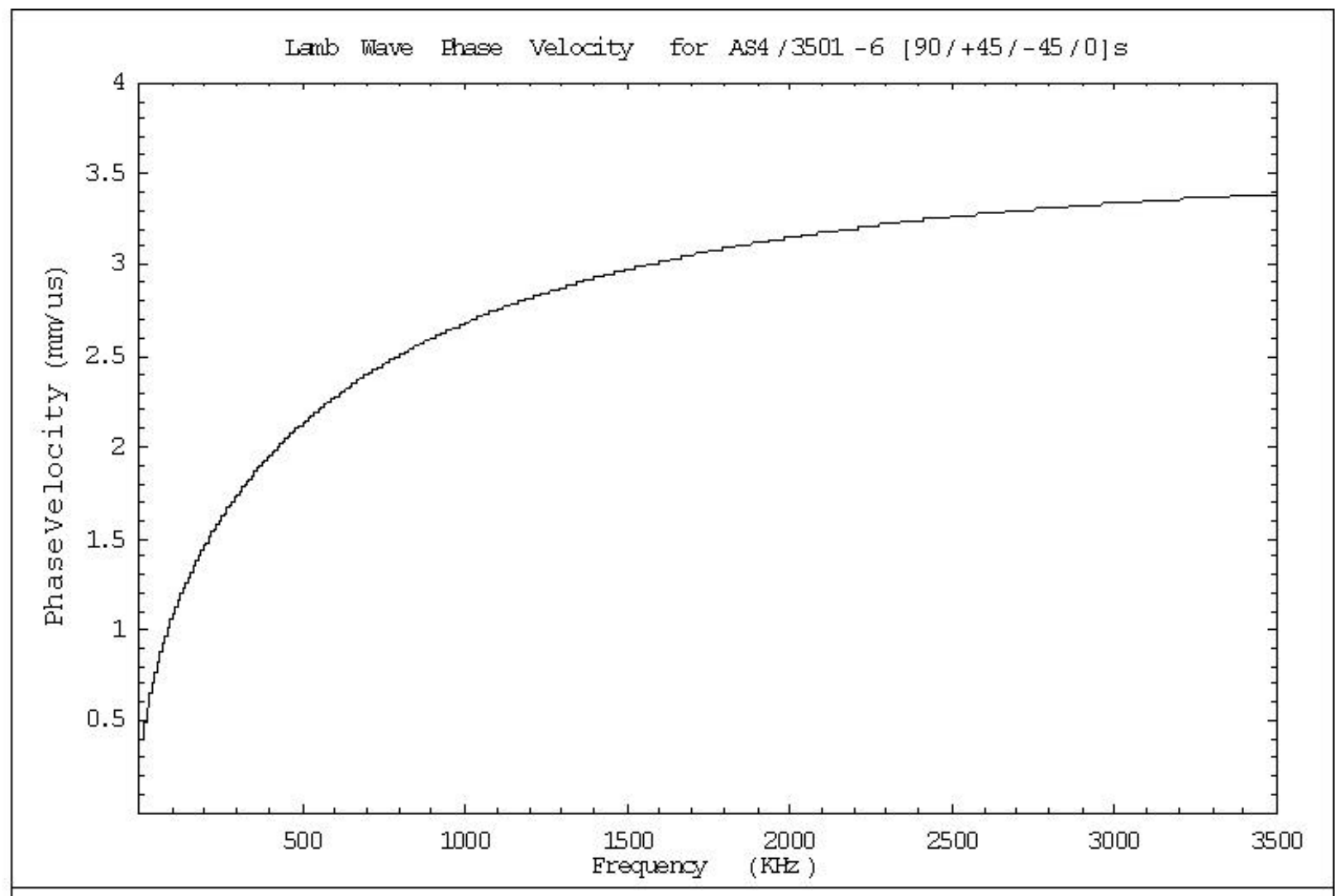

Figure 2: Phase velocity dispersion curve for the Ao mode of an 8-ply composite laminate 


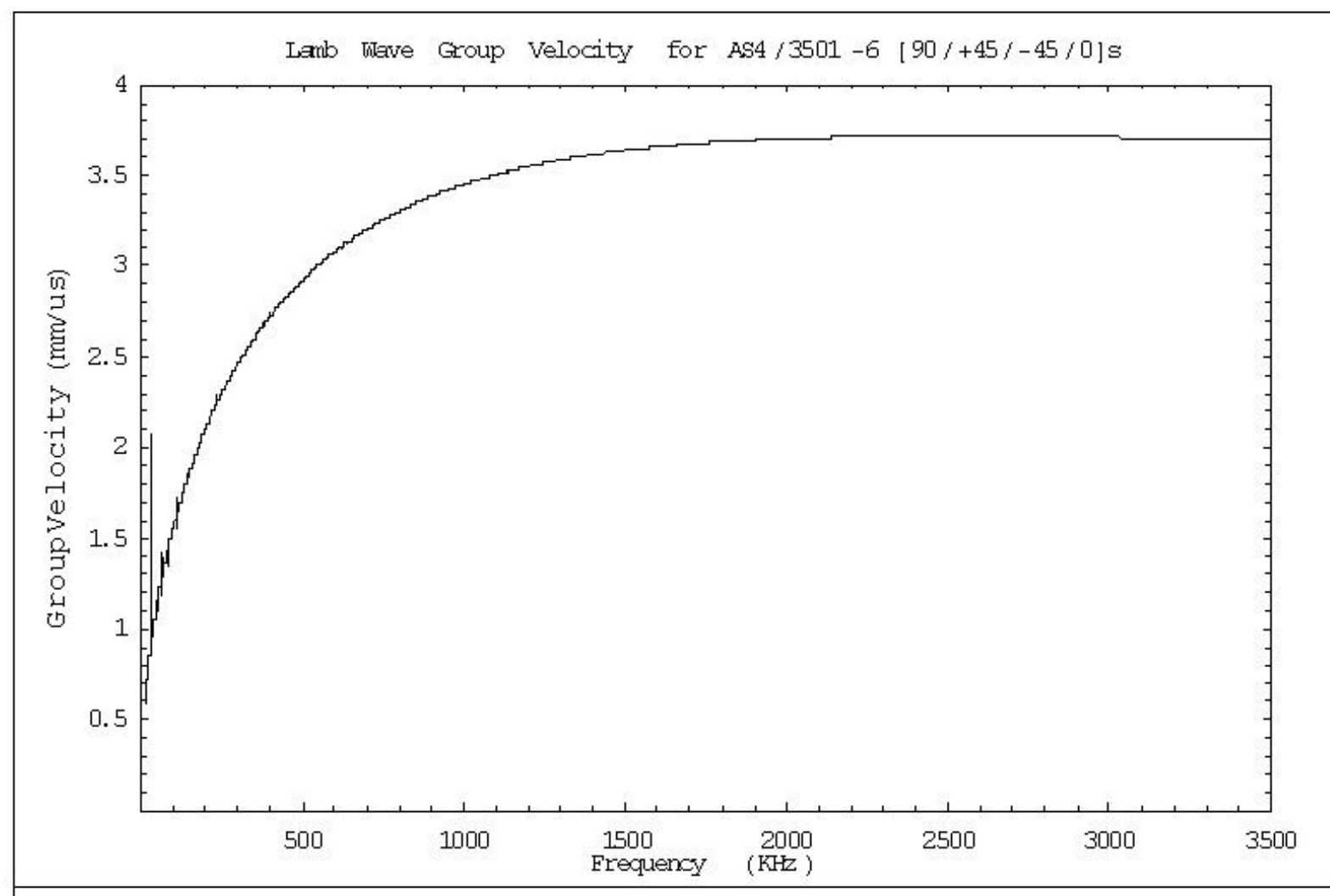

Figure 3: Group velocity dispersion curve for the Ao mode of an 8-ply composite laminate

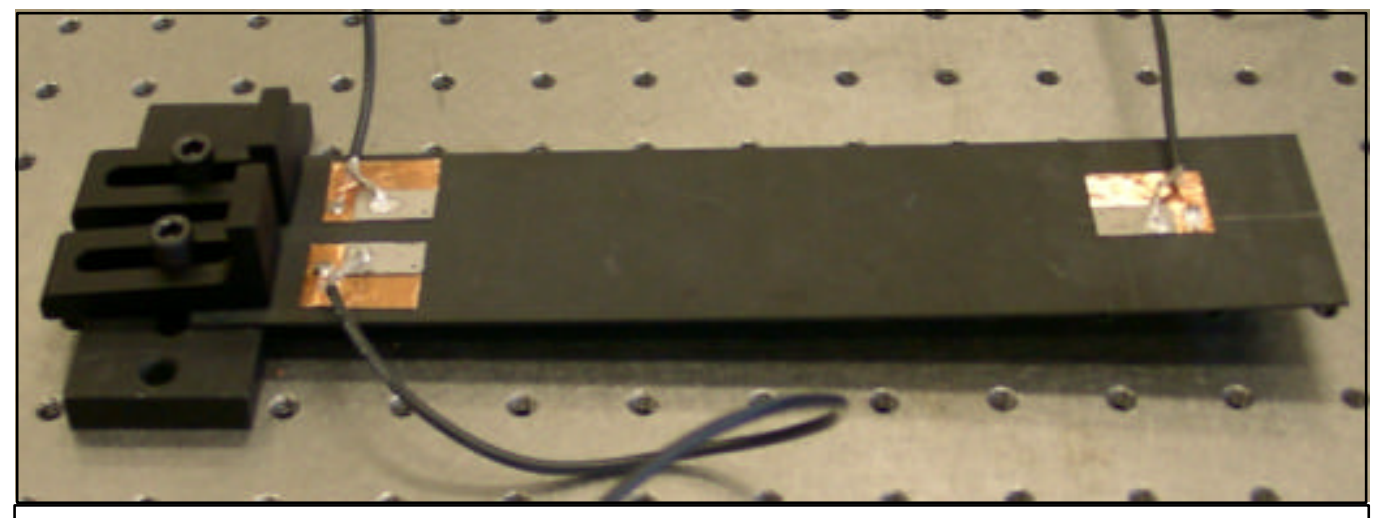

Figure 4: CFRP specimen $(250 \mathrm{~mm} \times 50 \mathrm{~mm})$ with piezoceramic actuator and sensors 


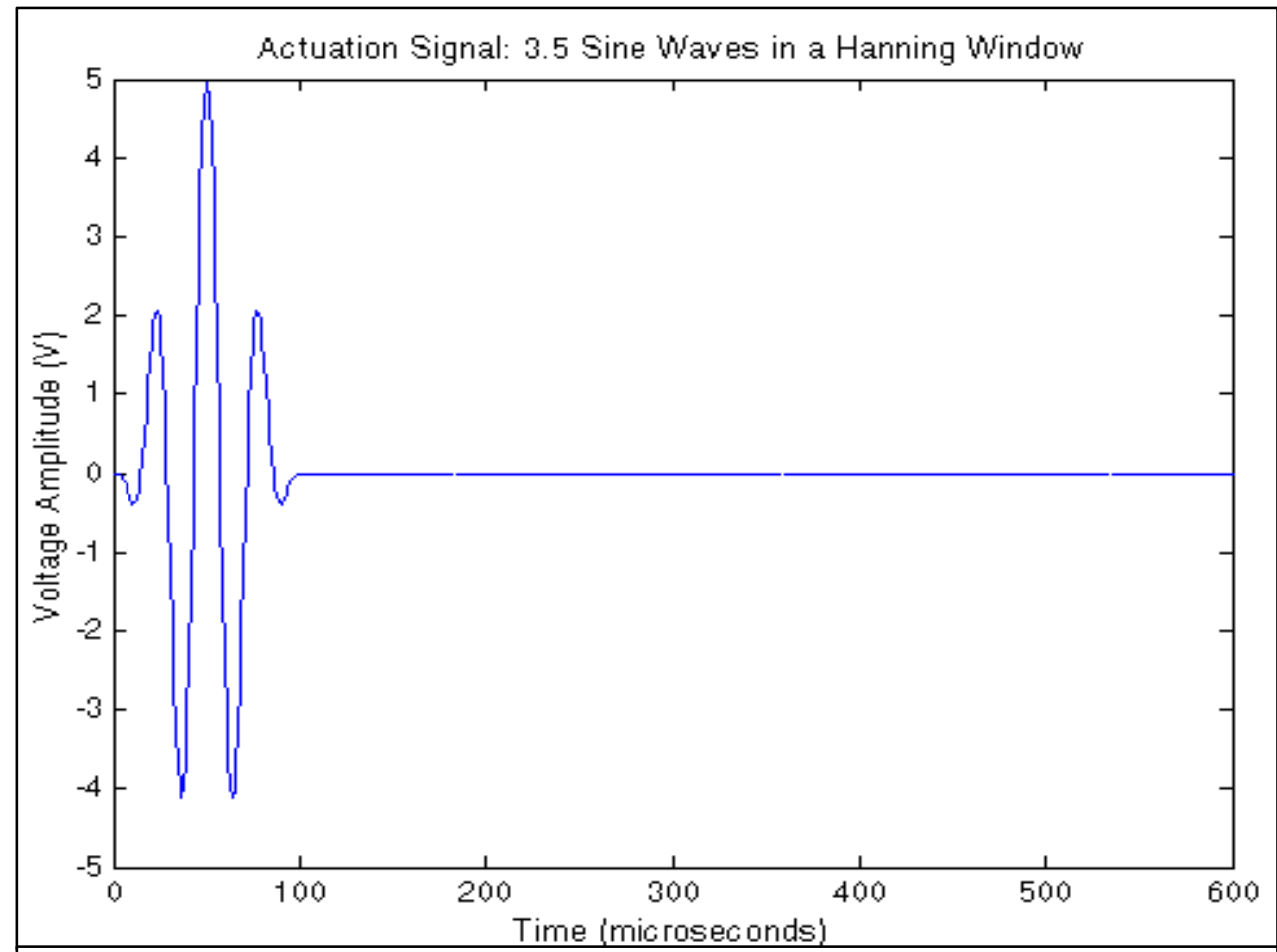

Figure 5: Actuation signal used to generate Ao Lamb mode, 3.5 sine waves at $15 \mathrm{kHz}$

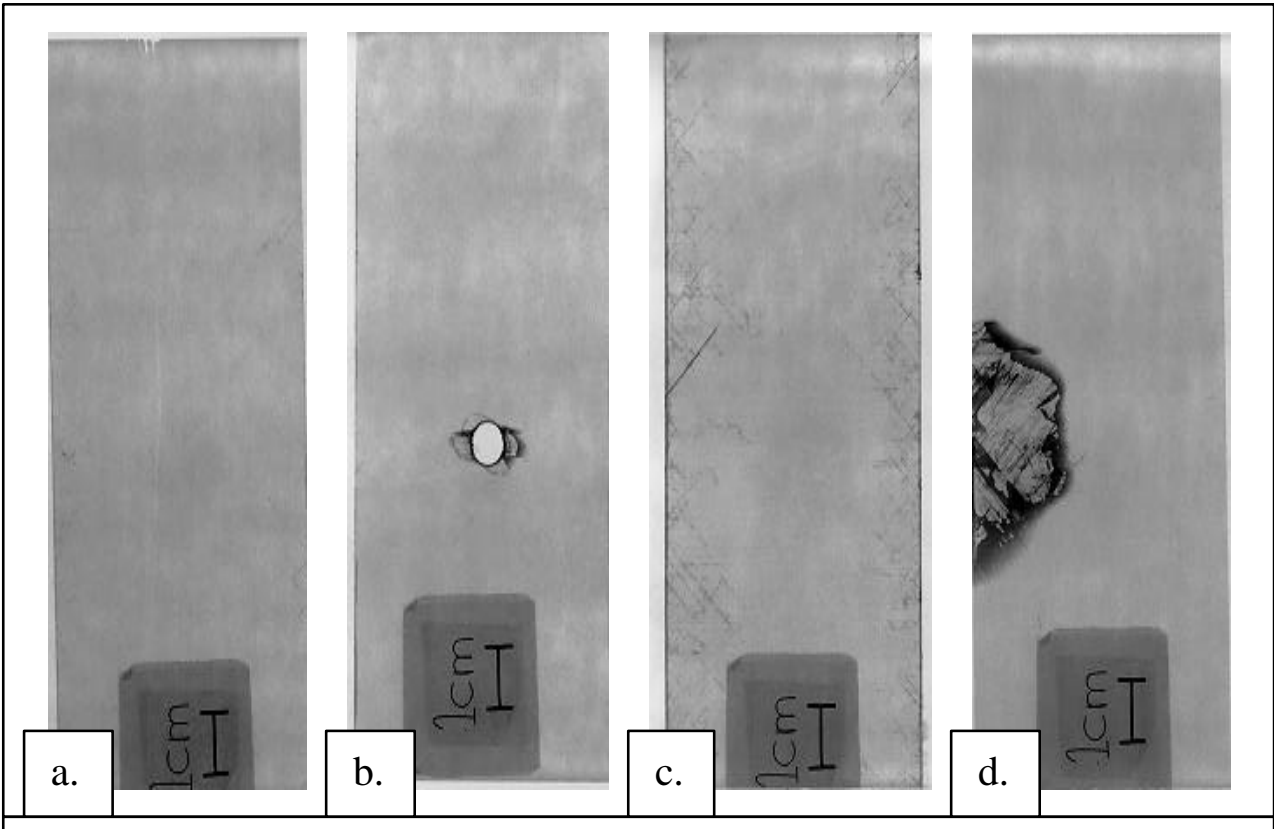

Figure 6: X-Radiographs of damaged specimens:

a. Control specimen with no damage present

b. Stress concentration specimen with drilled through-hole

c. Matrix-crack specimen with fatigue induced damage

d. Delamination specimen cut with a thin utility knife at the mid-plane 


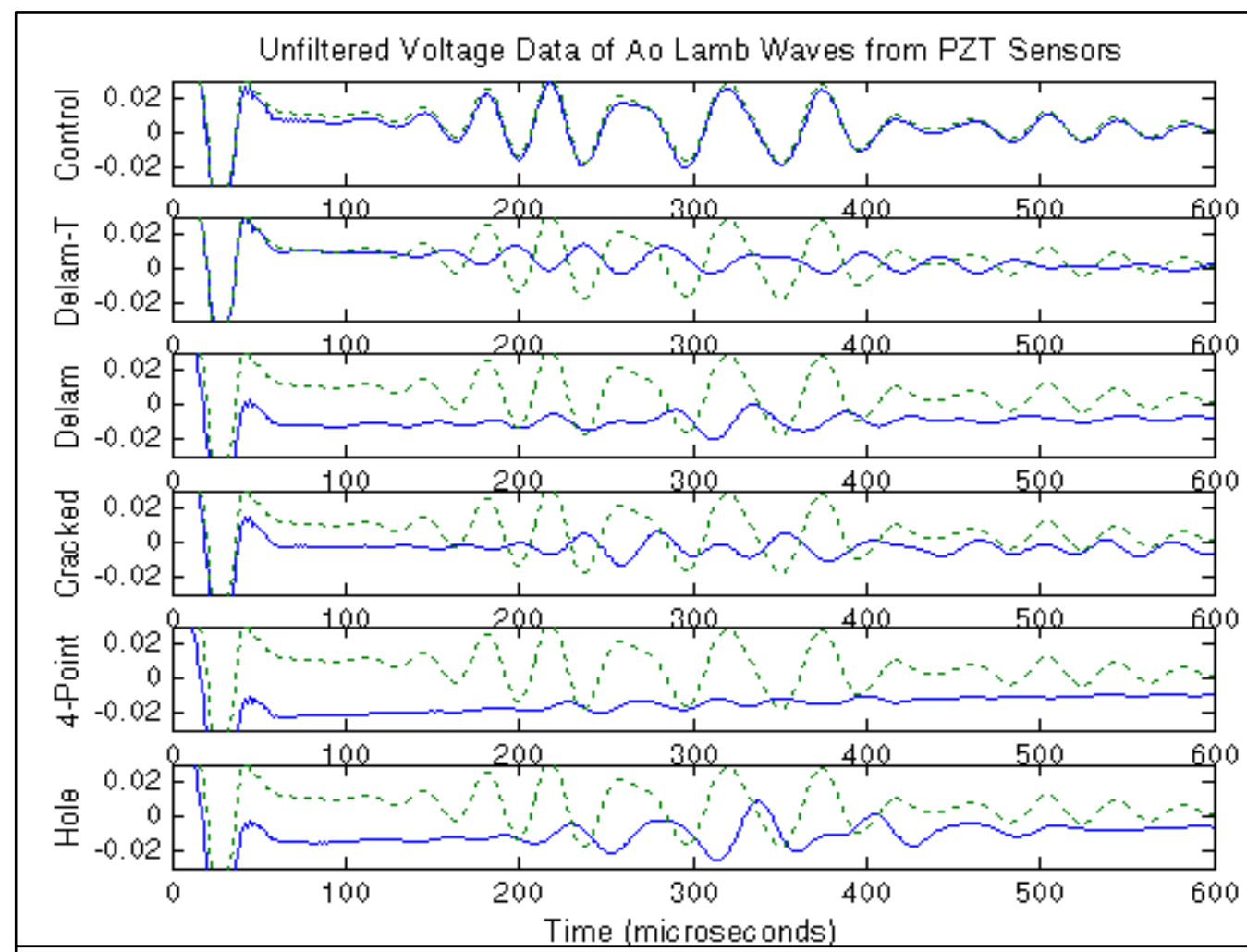

Figure 7: Time-trace of voltage signal from PZT sensor $20 \mathrm{~cm}$ from actuator, $15 \mathrm{kHz}$ signal Solid lines are damaged specimens; control is superimposed as a dashed line

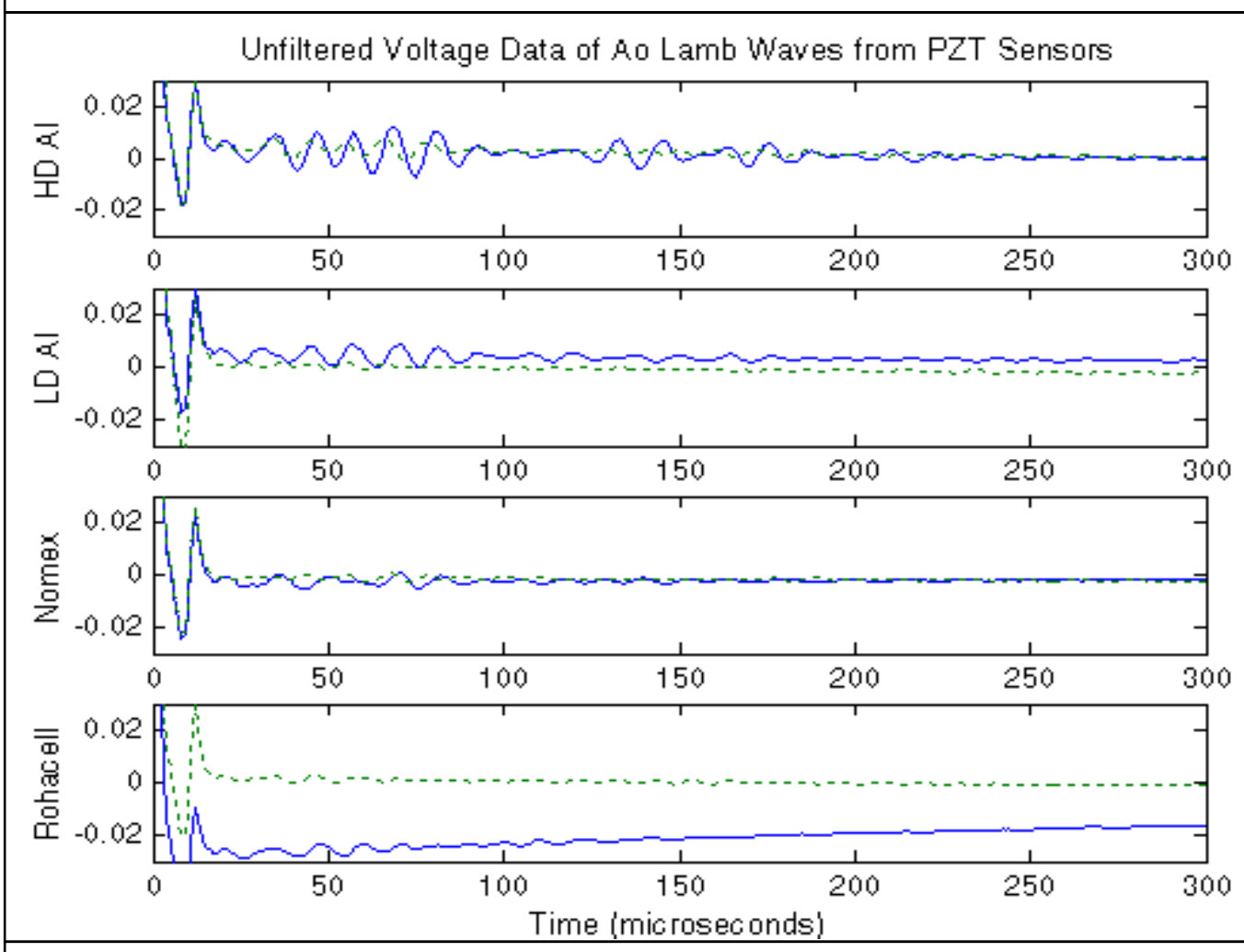

Figure 8: Time-trace of voltage signal from PZT sensor $20 \mathrm{~cm}$ from actuator, $50 \mathrm{kHz}$ signal Solid lines are undamaged beam controls, debonded specimens have dashed lines 


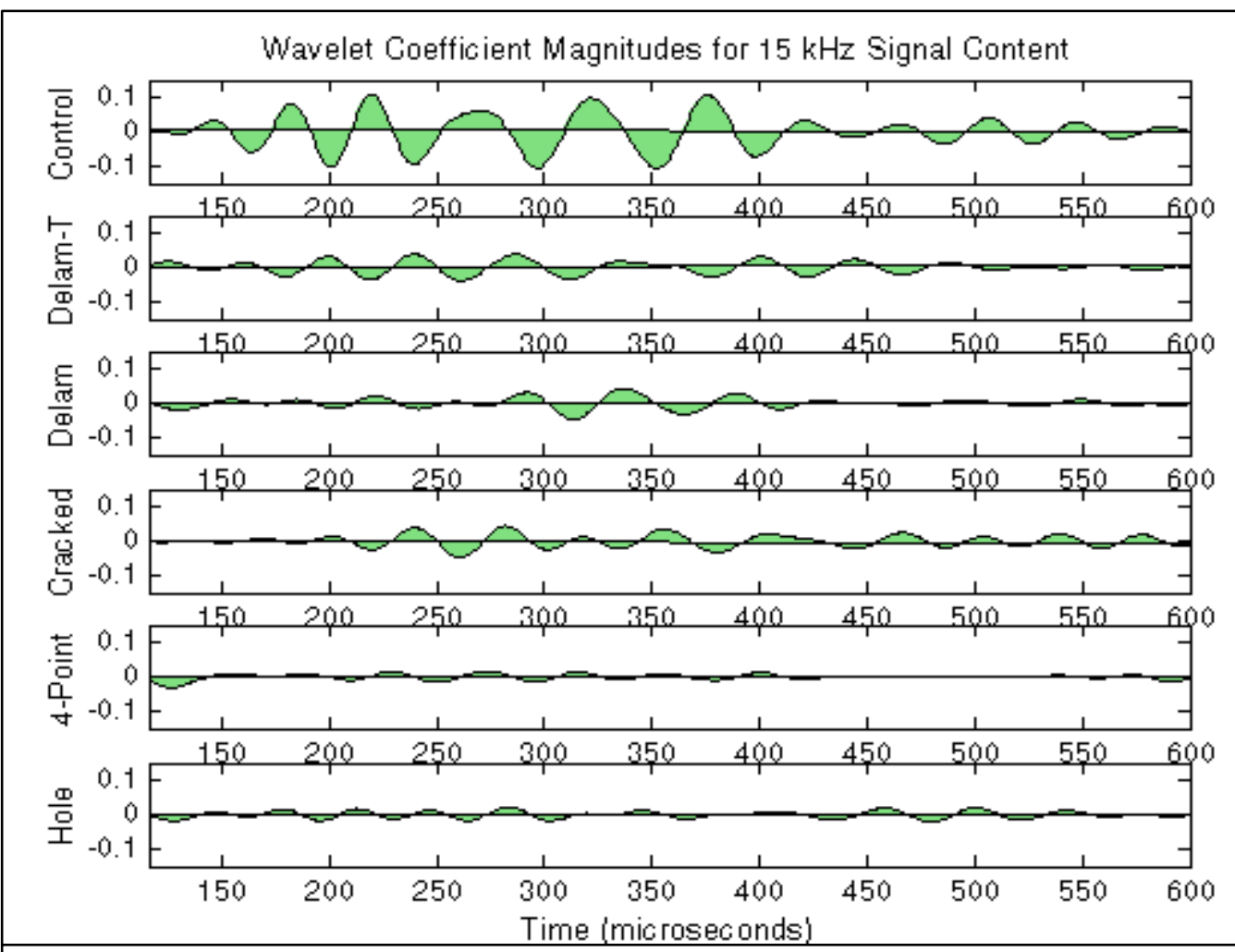

Figure 9: Wavelet coefficient plots for thin coupons; compares $15 \mathrm{kHz}$ energy content

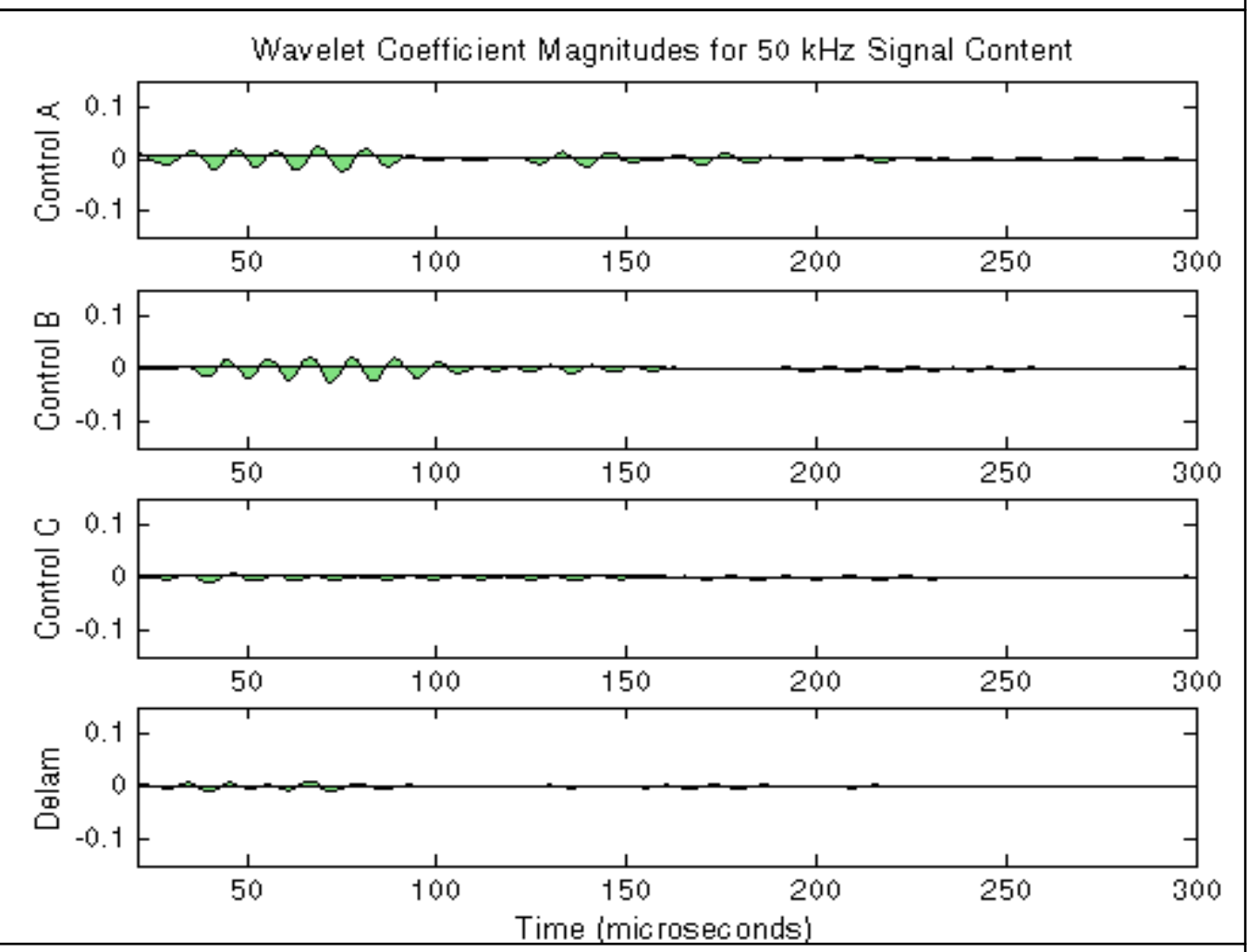

Figure 10: Wavelet coefficient plots for beam "blind test"; compares $50 \mathrm{kHz}$ energy content 